\title{
SIMULATION OF GAS FLOW OVER MICRO-SCALE AIRFOILS USING A HYBRID CONTINUUM-PARTICLE APPROACH
}

\author{
Quanhua Sun* and Iain D. Boyd ${ }^{\dagger}$ \\ Department of Aerospace Engineering \\ University of Michigan, Ann Arbor, MI, 48109
}

\begin{abstract}
Flow over micro-scale airfoils is investigated in order to understand the aerodynamic issues related to micro air vehicle design and performance. Studies have shown that such low Reynolds number flow exhibits rarefied phenomena and a flat plate having a thickness ratio of $5 \%$ has better aerodynamic performance than conventional streamlined airfoils. This paper simulates gas flows over a 5\% flat plate using a hybrid continuum-particle approach for flows having a Mach number of 0.2 and a Reynolds number varying between 1 and 200 . Investigation shows that low Reynolds number flows are viscous and compressible, and rarefied effects increase when the Reynolds number decreases. It is also found that there is a minimum lift slope for the flat plate airfoil at a Reynolds number near 10 and the drag coefficient monotonically increases with decreasing Reynolds number.
\end{abstract}

\section{Introduction}

There is increasing interest to design aircraft that are as small as possible for special military and civil missions (Mueller, 2001). Many such aircraft are currently under development, including micro-sized Unmanned Aerial Vehicles (UAVs) (http://www.fas.org/irp/program/collect/uav.htm) and micro Air Vehicles ( $\mu \mathrm{AVs}$ ) (McMichael and Francis, 1997). However, research and development at significantly smaller scales is still in its infancy. There are very few computations and experiments for aerodynamics of airfoils at low Reynolds numbers (Re) below 1,000. The objectives of this paper are to understand external flows at low Reynolds numbers and to investigate the aerodynamics of a micro-scale airfoil at Reynolds numbers below 1,000 .

The dependence of airfoil performance on the flow Reynolds number is well known. When $\operatorname{Re}>10^{6}$, the variation of airfoil characteristics with Reynolds number is rather slow (Jones, 1990). However, when the Reynolds number is on the order of $10^{5}$, the aerodynamics of airfoils varies rapidly with the configuration of airfoils. Figure 1 shows the experimental results of Schmitz adapted from Jones (1990) for a thin flat plate and a conventional shaped airfoil. Here the thin flat plate is inferior to the shaped airfoil when $\mathrm{Re}=1.2 \times 10^{5}$, but is superior to the same shaped airfoil when $\operatorname{Re}=4 \times 10^{4}$. Sunada et

* Graduate Student Research Assistant, Student Member AIAA

$\uparrow$ Professor, Associate Fellow AIAA

al. (1997) compared the aerodynamics of several airfoils when $\mathrm{Re}=4 \times 10^{3}$ by towing airfoils through water in a tank. They concluded that a flat plate with a thickness ratio of $5 \%$ has larger lift slope than conventional streamlined airfoils as shown in Figure 2.
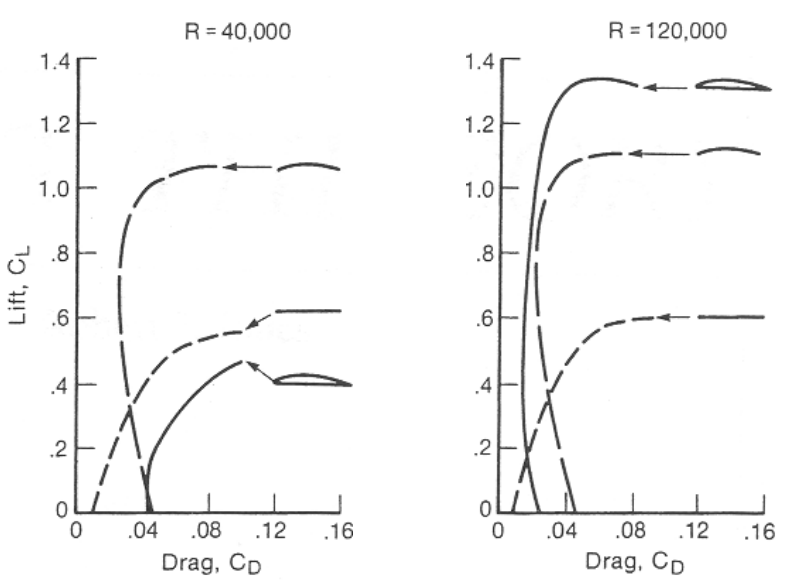

Figure 1. Airfoil shapes at different Reynolds numbers.

airfoil shape

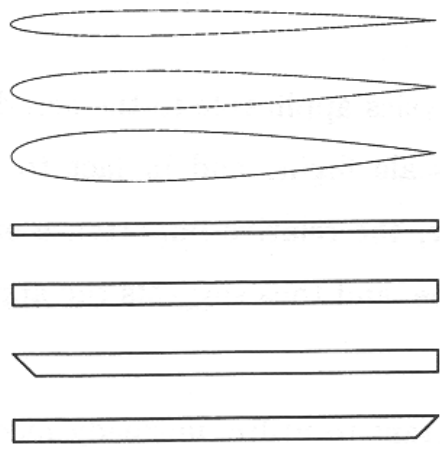

Figure 2. Comparison of lift slope of airfoils at a Reynolds number of 4,000, after Sunada et al. (1997)

It is also found that the properties of flows at small scales depend on the Mach number (M). The drag on a micro-scale flat plate is shown in Figure 3 (Sun, 2003), which clearly demonstrates the compressible effects for low Reynolds 
number flows. The results were obtained using a numerical technique called the information preservation (IP) method (Sun and Boyd, 2002), and agreed very well with experimental data obtained by Schaaf and Sherman (1954).

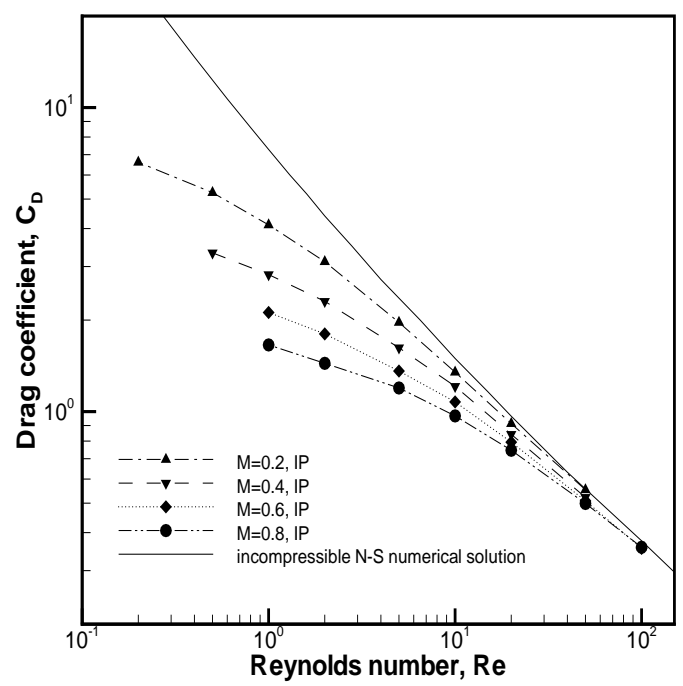

Figure 3. Drag on a flat plate depends on Reynolds number and Mach number as predicted by the IP method.

Low Reynolds number flows are dominated by the viscous effects and compressible effects, which also suggests that rarefied effects exist in these flows because an important rarefied parameter, the Knudsen number $(\mathrm{Kn})$, is a combination of the Reynolds number and the Mach number. Therefore, numerical simulation of low Reynolds number flows is challenging. Continuum equations, e.g., the Navier-Stokes equations, are physically invalid for rarefied gas flows, and so methods based on kinetic theory must be used for modeling low Reynolds number flows.

In this paper, we simulate gas flows over a $5 \%$ flat plate and investigate the aerodynamics of this flat plate airfoil for Reynolds numbers in the range of $1<\operatorname{Re}<200$ when the free stream Mach number is 0.2 using a hybrid continuum-particle approach. The rest of the paper is organized as follows. Section 2 describes the numerical approach. Section 3 presents the numerical results and related experimental and theoretical data. At the end, conclusions are given in Section 4.

\section{Numerical approach}

Low Reynolds number flows exhibit rarefied effects as discussed in the previous section. Therefore, continuum equations are not physically accurate, and kinetic methods are required. However, kinetic methods are generally several orders of magnitude more numerically expensive than continuum computational fluid dynamic techniques. It is the goal to apply a kinetic method only when it is required for a simulation. Namely, an efficient approach is to combine the numerical efficiency of continuum approaches for the continuum region and the physical accuracy of a kinetic approach for the rarefied region.

The hybrid approach used for the current investigation is a hybrid continuum-particle approach (Sun et al., 2002) that combines a Navier-Stokes approach and the information preservation (IP) method. The entire computational domain is separated into continuum domains and particle domains by an interface, which is adaptively determined by a continuum breakdown parameter. The Navier-Stokes approach is used in the continuum domain and the IP method is applied to the particle domain, while necessary information is exchanged through the interface at every time step.

\subsection{The Navier-Stokes approach}

The Navier-Stokes equations are expressed in conservation form as follows:

$$
\frac{\partial \mathbf{U}}{\partial t}+\nabla \cdot\left(\mathbf{F}_{o}+\mathbf{F}_{\mu}\right)=0
$$

where

$$
\begin{gathered}
\mathbf{U}=\left[\begin{array}{c}
\rho \\
\rho \mathbf{u} \\
e
\end{array}\right], \mathbf{F}_{o}=\left[\begin{array}{c}
\rho \mathbf{u} \\
\rho \mathbf{u u}+p \mathbf{I} \\
(e+p) \mathbf{u}
\end{array}\right], \mathbf{F}_{\mu}=\left[\begin{array}{c}
0 \\
-\boldsymbol{\tau} \\
-\boldsymbol{\tau} \cdot \mathbf{u}+\mathbf{q}
\end{array}\right] \\
p=(\gamma-1) \rho \varepsilon, \varepsilon=e / \rho-\mathbf{u} \cdot \mathbf{u} / 2 \\
\tau_{i j}=\mu\left(\frac{\partial u_{i}}{\partial x_{j}}+\frac{\partial u_{j}}{\partial x_{i}}\right)-\frac{2}{3} \mu \frac{\partial u_{k}}{\partial x_{k}} \delta_{i j}, q=-\kappa \nabla \cdot T
\end{gathered}
$$

In the previous expressions, $\rho$ is the mass density, $\mathbf{u}$ is the velocity vector, $p$ is the pressure, and $e$ is the specific total energy. In order to match the variable hard sphere (VHS) model (Bird, 1994) used in the IP method, the viscous coefficient $\mu$ and the coefficient of thermal conductivity $\kappa$ are expressed as follows:

$$
\begin{aligned}
& \mu=\mu_{0}\left(\frac{T}{T_{0}}\right)^{\omega} \\
& \kappa=\frac{9 \gamma-5}{4 \gamma-4} R \mu
\end{aligned}
$$

where $\mu_{0}$ is the viscous coefficient of the gas at temperature $T_{0}, \omega$ is the viscosity temperature index in the VHS molecular model, and $R$ is the specific gas constant.

The continuum approach employed in the hybrid approach solves the Navier-Stokes equations using a finite volume formulation. The fluxes are evaluated with a second-order accurate modified Steger-Warming flux-vector splitting approach (MacCormack and Candler, 1989). Slip boundary conditions are implemented with the use of the Maxwell-type slip velocity expression (Schaaf and Chambre, 1958):

$$
u_{s}=\left.\frac{2-\sigma}{\sigma} \lambda \frac{\partial u}{\partial n}\right|_{w}
$$

where the mean free path $\lambda$ is given by $\lambda=2 \mu / \rho\langle c\rangle$ and $\langle c\rangle$ is the mean molecular speed of the gas. 


\subsection{The information preservation method}

The information preservation (IP) method (Fan and Shen, 2001, Sun and Boyd, 2002) is a recently developed particle method for modeling rarefied gas dynamics based on the direct simulation Monte Carlo (DSMC) method (Bird, 1994).

The DSMC method models a gas flow with representative particles in simulated physical space. The position coordinates and the velocity components (and the internal energy state) of simulated particles are preserved and are modified with time as the particles are concurrently followed through representative collisions and boundary interactions. The IP method additionally preserves flow information at the macroscopic level for the simulated particles and for the computational cells in order to reduce the statistical scatter associated with particle methods. The preserved information at the macroscopic level is modeled at each time step, and is sampled to output the flow field. It is found that the IP method exhibits very small statistical scatter for flow properties, and thus is less numerically expensive than the DSMC method especially for low-speed gas flows. The validity of the IP method has been shown by many examples (Sun, 2003), including typical subsonic steady flows and low frequency unsteady flows ranging from the near-continuum regime to the free molecular regime.

\subsection{The hybrid continuum-particle approach}

The hybrid approach couples the Navier-Stokes approach and the information preservation method through an interface. In order to set the continuum domain as large as possible, the interface can be determined by a continuum breakdown parameter. The parameter $B$ suggested by Garcia et al. (1999) is implemented in the hybrid code as the continuum breakdown parameter. However, there is no theory that indicates the cutoff value for the continuum breakdown parameter. Therefore, a small number is used to set as the cutoff value. The cutoff value for the parameter $B$ is set as 0.005 in the implementation of the hybrid approach.

Once the interface is determined, the Navier-Stokes approach is used for the continuum domain while the IP method is applied to the particle domain. However, the NavierStokes solver requires the information from the particle domain to calculate the flux on the interface, and the IP solver needs the interface to provide incoming particles. Because the IP method preserves information at the macroscopic level for the computational cells, the Navier-Stokes solver can treat particle cells as ghost cells. Then the interface becomes internal cell edges for the continuum solver. In order to avoid directly generating particles through the interface for the particle solver, buffer cells and reservoir cells are used in the continuum domain as shown in Figure 4. Particles are regenerated in the reservoir cells based on the information from the continuum solver using the first-order Chapman-Enskog distribution (Garcia and Alder, 1998) at every time step. Buffer cells are used because they can improve the represented information of particles that can enter the particle domain through the interface.

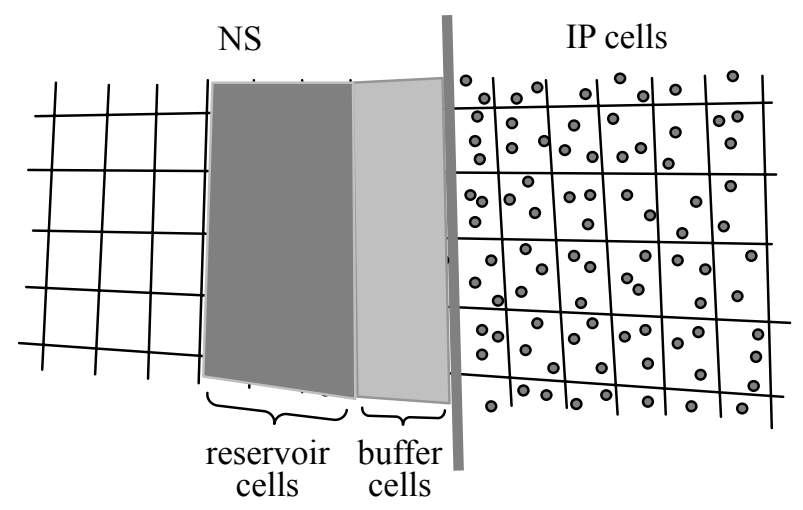

Figure 4. Illustration of the interface and cell structure for the hybrid continuum-particle approach.

\section{Numerical simulation of gas flows over a micro- scale airfoil}

Gas flows over a micro-scale airfoil at low Reynolds numbers are simulated using the hybrid continuum-particle approach. The airfoil is a 30 -micron-long flat plate having a thickness ratio of $5 \%$. The plate is kept at a fixed temperature of $295 \mathrm{~K}$, and full thermal accommodation is assumed. The free air stream has a Mach number of 0.2 and a temperature of 295K. Three free stream pressures are considered in this investigation: $1.0 \mathrm{~atm}, 0.1 \mathrm{~atm}$, and $0.01 \mathrm{~atm}$. The corresponding Reynolds numbers are 135.7, 13.57, and 1.357. The VHS molecular model (Bird, 1994) is used for the simulations.

The computational domain has an area with a radius of 150 microns. The free stream boundary condition is implemented

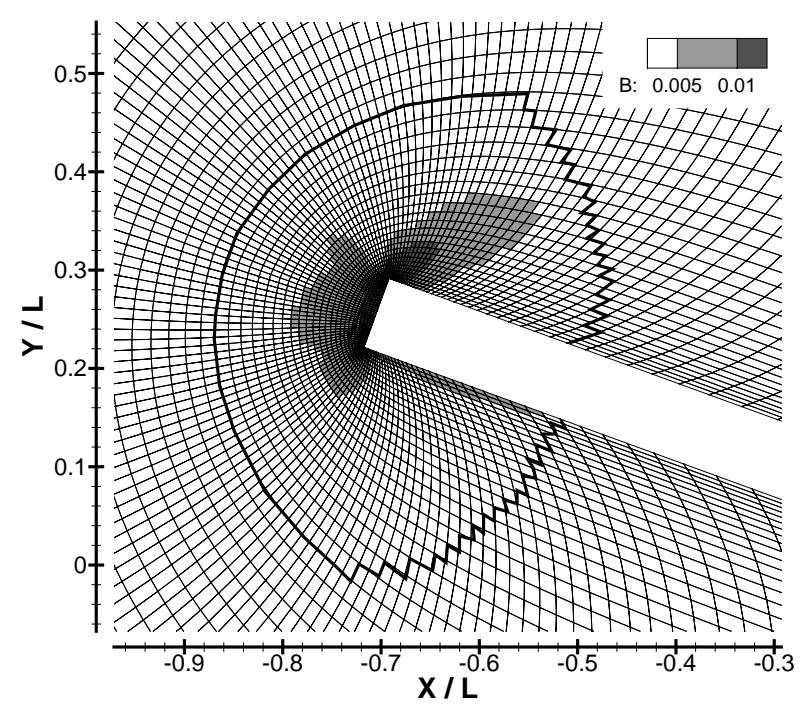

Figure 5. Part of the computational grid for flow over a $5 \%$ flat plate having an angle of attack of $20^{\circ}$ when $\mathrm{Re}=135.7$. 
using the characteristic line method (Hirsh, 1991). The computational cells are clustered around the flat plate, especially near the leading edge and the trailing edge. The cell size of cells near the plate is less than the mean free path of air, while the size of cells far from the plate can be much larger because the gradients of local flow properties are large. However, the cell size of continuum cells does not need to be less than the mean free path of air. Part of the computational grid is shown in Figure 5 for the case when $R e=135.7$ and the angle of attack $\alpha=20^{\circ}$. The total number of computational cells is 21,300 when $\mathrm{Re}=135.7,14,200$ when $\mathrm{Re}=13.57$, and 5,100 when $\operatorname{Re}=1.357$.

When the Reynolds number is 135.7 , the computational domain is divided by a fixed interface, and the particle domain is larger than that indicated by the continuum breakdown parameter $B$ with the cutoff value of 0.005 . For example, the particle domain in Figure 5 is the domain surrounded by the interface as indicated by the thicker solid line. When the Reynolds number is smaller, the hybrid approach is only used for the flow to reach a steady state, and thereafter the entire computational domain is simulated using the IP method.

\subsection{Viscous effects}

The Reynolds number denotes the ratio of the inertia force to the viscous force. Hence, the viscous effects are important when the Reynolds number of a flow is small. As a result, the leading and trailing edges affect a significant fraction of the plate length, and the boundary layer thickness is very large.

The velocity contour for $|V| / V_{\infty}=0.8$ is shown in Figure 6 for all three cases when the angle of attack is zero. Clearly, when the Reynolds number is small, the flat plate slows the flow because of the gas viscosity, and this effect increases when the flow Reynolds number decreases. The parallel velocity profiles of the flow above the flat plate for three cases is shown in Figure 7 when the angle of attack is zero. In Figure 7 , the velocity scale is the same for the three cases. When the Reynolds number is 135.7 , the velocity profile shows a typical boundary layer. However, the thickness of the boundary layer becomes very large for the cases with lower Reynolds number if the concept of the boundary layer can still be used.

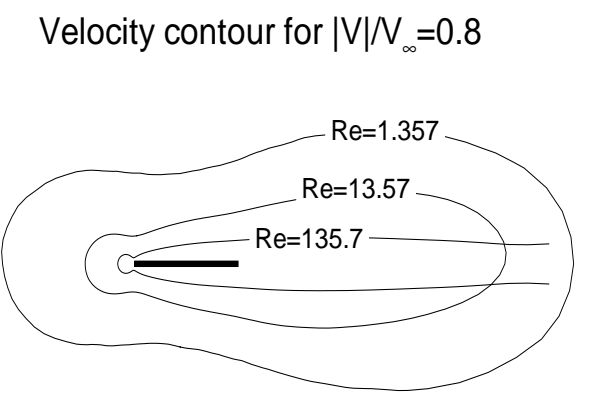

Figure 6. Reynolds number effects on a specific velocity contour for gas flows over a $5 \%$ flat plate airfoil.
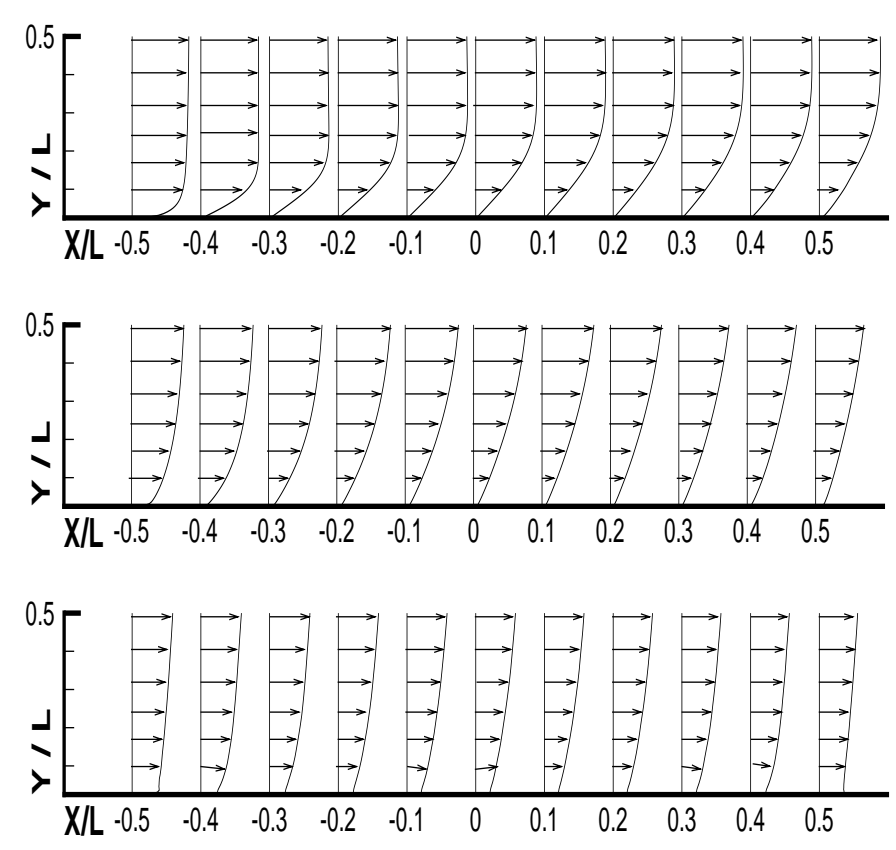

Figure 7. Parallel velocity profiles above the flat plate when the angle of attack is zero at various Reynolds numbers (upper: $\mathrm{Re}=135.7$; middle: $\mathrm{Re}=13.57$; lower: $\mathrm{Re}=1.357$ ).

\subsection{Compressible effects}

It is generally assumed that a flow is incompressible when the flow Mach number is below 0.3. However, a flow becomes compressible at a low Reynolds number even when the Mach number of the flow is small. Figure 8 shows the pressure coefficient distribution on the lower side of the flat plate when the angle of attack is $20^{\circ}$. It is found that the pressure coefficient for the case with $\mathrm{Re}=13.57$ is very close to that when $\mathrm{Re}=135.7$, which may indicate the compressible effect is

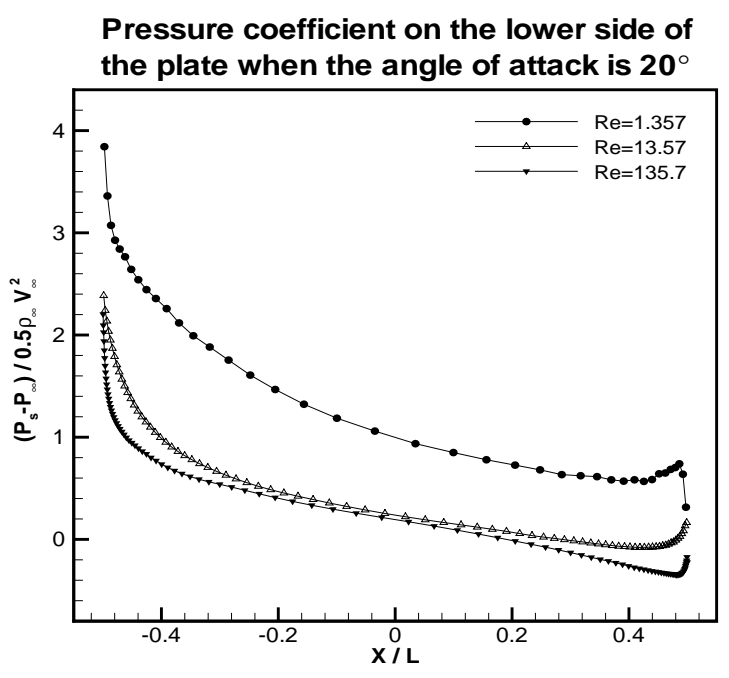

Figure 8. Reynolds number effect on the compressibility for flows over a $5 \%$ flat plate airfoil. 
not significant when the Reynolds number is above 10 . However, there are very large differences between the pressure coefficients when the Reynolds number is 1.357 and 13.57. This is not surprising because free molecular theory predicts an even larger pressure coefficient.

\subsection{Velocity slip}

A slip velocity exists for flows in the slip regime where the Knudsen number (the ratio of the mean free path of the gas to the smallest characteristic length of the flow) is between 0.01 and 0.1 . However, this Knudsen number range is only conceptional because the smallest characteristic length is not well defined.

The slip velocity on the upper side of the plate is shown in Figure 9 for all three cases when the angle of attack is zero. Although the global Knudsen number based on plate length is only about 0.002 when the Reynolds number is 135.7 , there is a considerable amount of velocity slip near the leading edge. It is also found that the slip velocity increases when the Reynolds number decreases. These results show that the rarefied effect exists only near the leading edge when the Reynolds number is not too small and the effect expands to larger domains when the Reynolds number decreases.

\section{Slip velocity on the upper side of the plate}

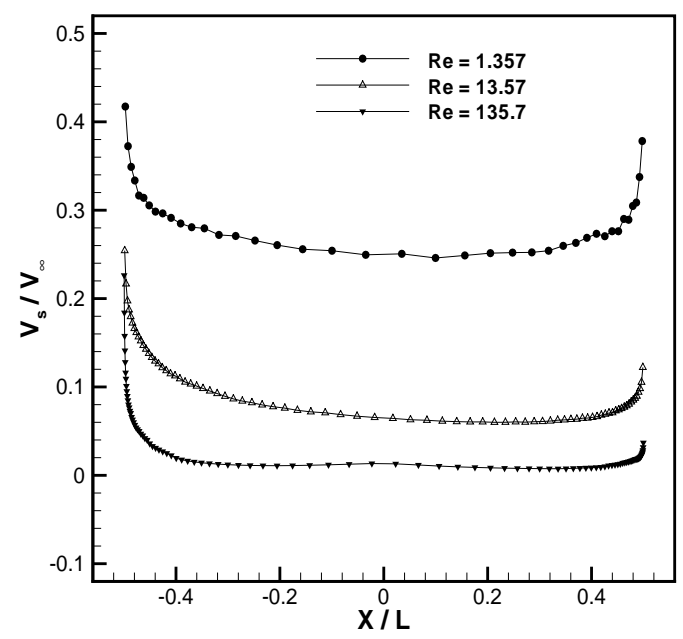

Figure 9. Reynolds number effect on the slip velocity.

The friction coefficient on the upper side of the plate is shown in Figure 10. The zigzag result when $\mathrm{Re}=1.357$ is due to the statistical scatter associated with the particle approach. Clearly, the friction coefficient increases when the flow Reynolds number decreases. This is consistent with the slip velocity shown in Figure 9 because the friction coefficient can be expressed in terms of the slip velocity as follows:

$$
C_{f}=\frac{\langle c\rangle}{V_{\infty}} \cdot \frac{V_{s}}{V_{\infty}}
$$

It is interesting to see whether the linear expression $\tau=\mu \partial u / \partial y$ is still valid. Figure 11 compares the friction
Friction coefficient on the upper side of the plate

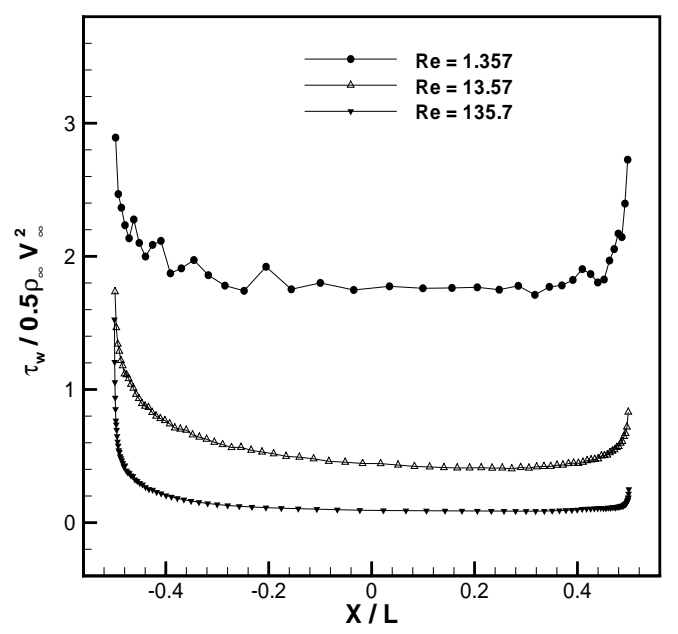

Figure 10. Reynolds number effect on the friction coefficient.

coefficient obtained from this expression and that directly from the IP simulation. It shows that the linear expression gives larger shear stress, which may mean that continuum equations are invalid when $\mathrm{Re}=13.57$, especially near the leading edge and the trailing edge.

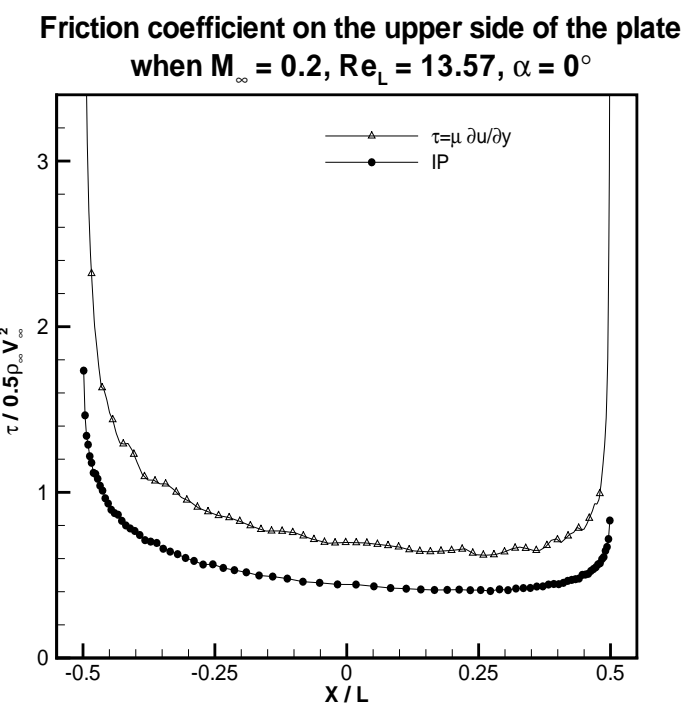

Figure 11. Comparison of skin friction on the flat plate obtained using a linear expression and from the particle simulation.

\subsection{Flows at various angles of attack}

The flow patterns when $\mathrm{Re}=135.7$ are illustrated in Figure 12 under different angles of attack. The figure shows the pressure flow field and some streamlines around the airfoil. Clearly, the pressure increases where the flow faces the airfoil, and drops where the flow leaves the airfoil. There is no flow separation when the angle of attack $(\alpha)$ is equal to or less than $10^{\circ}$. However, when $\alpha=20^{\circ}$, the flow begins to separate near the upper leading edge because of the strong local adverse 

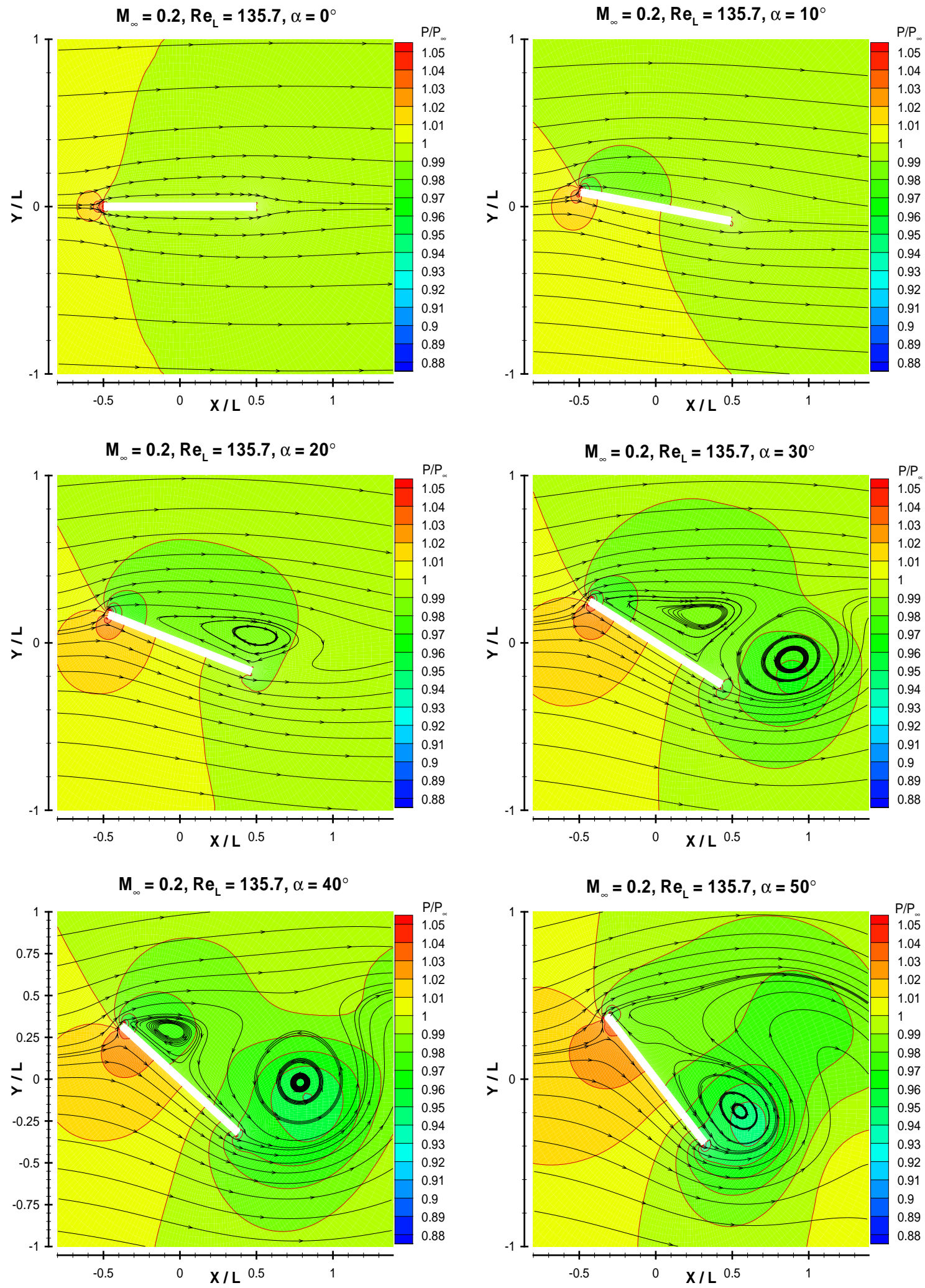

Figure 12. Pressure flow field and streamlines for flows over a $5 \%$ flat plate when $\mathrm{M}_{\infty}=0.2, \mathrm{Re}_{\mathrm{L}}=135.7$, with the angle of attack ranging from $0^{\circ}$ to $50^{\circ}$. 

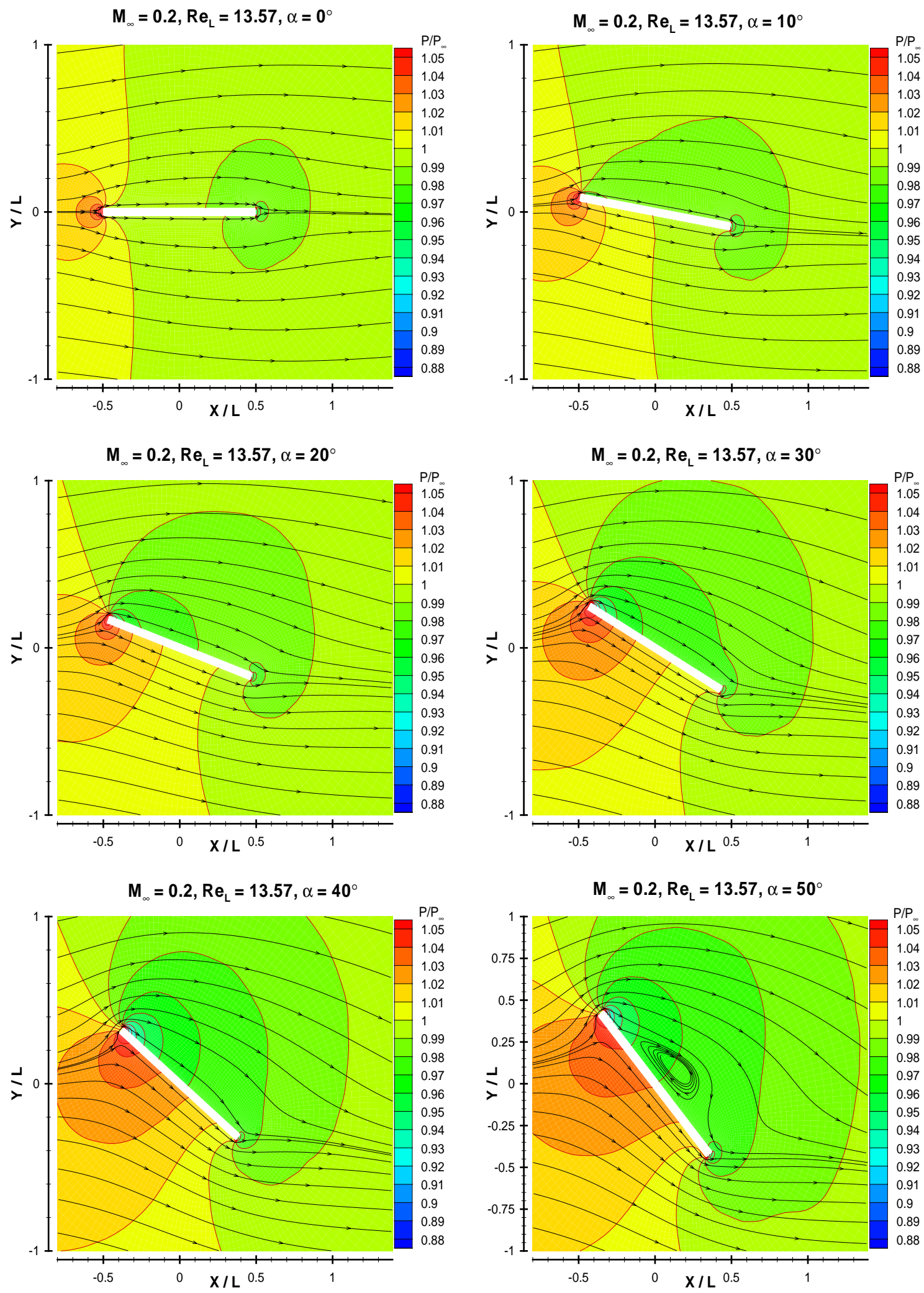

Figure 13. Pressure flow field and streamlines for flows over a $5 \%$ flat plate when $\mathrm{M}_{\infty}=0.2, \operatorname{Re}_{\mathrm{L}}=13.57$, with the angle of attack ranging from $0^{\circ}$ to $50^{\circ}$. 
pressure gradient. When $\alpha=30^{\circ}$, another separation occurs near the trailing edge of the plate. The flow is then dominated by the two separation regions or two vortices. The downstream vortex becomes stronger when the angle of attack increases $\left(\alpha=40^{\circ}\right)$, and this vortex almost eats away the upstream vortex when $\alpha=50^{\circ}$.

The flow patterns when $\mathrm{Re}=13.57$ are illustrated in Figure 13. Compared to the flows when $\mathrm{Re}=135.7$, these flows at the lower Reynolds number exhibit different flow behavior. Except for the phenomena that are already mentioned, there are several new effects. First, flow separation is delayed and weakened when the Reynolds number decreases. There is no separation when $\mathrm{Re}=1.357$ for the angle of attack up to $50^{\circ}$ (figures are not shown here; refer to Sun, 2003). Second, the pressure gradient near the leading edge decreases although the overall pressure variation increases when the flow Reynolds decreases. Therefore, it can be anticipated that the aerodynamic characteristics of the micro-scale airfoil at low Reynolds number flows are very different from those at high Reynolds numbers.

\subsection{Aerodynamic characteristics of the $5 \%$ flat plate at low Reynolds numbers}

The airfoil theory for inviscid flow indicates that the lift slope for a thin airfoil is $2 \pi$. However, the lift slope decreases when the flow Reynolds number decreases for conventional streamlined airfoils (Sunada et al., 1997). In this subsection, we compare the aerodynamics of a $5 \%$ flat plate from experiments, IP simulations, and free molecular theory.

Sunada et al. (1997) conducted experiments on a 5\% flat plate at the chord Reynolds number of 4,000 by towing the airfoil through water in a tank. We plot their experimental data in Figure 14. It was estimated that the experiment error is within

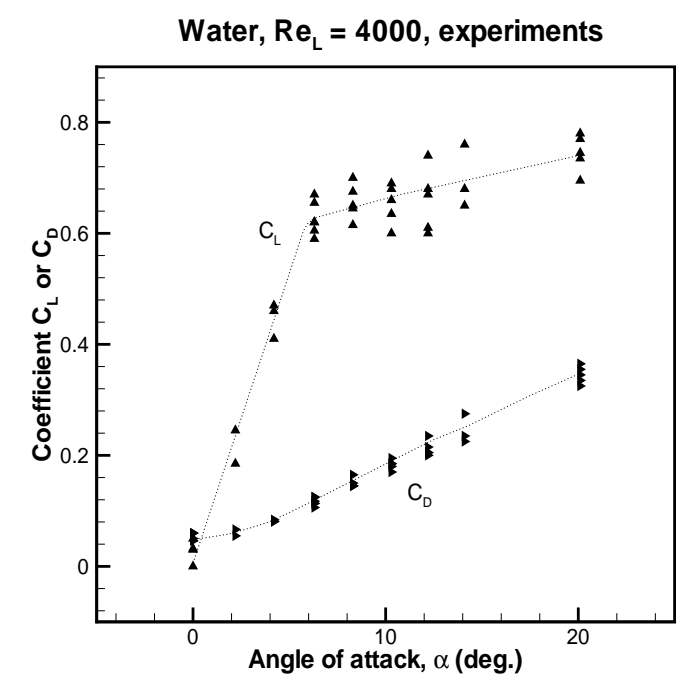

Figure 14. Measured drag coefficient and lift coefficient after Sunada et al. (1997) when the Reynolds number is 4,000.
18 percent for the lift and drag coefficients. The figure shows the lift slope is 5.8 and the drag coefficient is less than 0.1 at small angle of attack.

The lift and drag coefficients calculated for the current simulations are plotted in Figures 15-17. Figure 15 shows the results when $\mathrm{Re}=135.7$. The lift slope is about 3.0 and the drag coefficient is roughly 0.4 at small angle of attack. The lift does not increase linearly because the flow is separated when the angle of attack is $20^{\circ}$ or larger. Figure 16 shows the results when $\mathrm{Re}=13.57$. Here, the lift slope is about 2.8 and the drag coefficient is a little larger than 1 at small angle of attack. In addition, the ratio of lift to drag is less than 1 because of the large drag coefficient. Figure 17 shows the results when $\mathrm{Re}=1.357$. It is found that the lift slope is about 4.2 and the drag coefficient is larger than 4 .

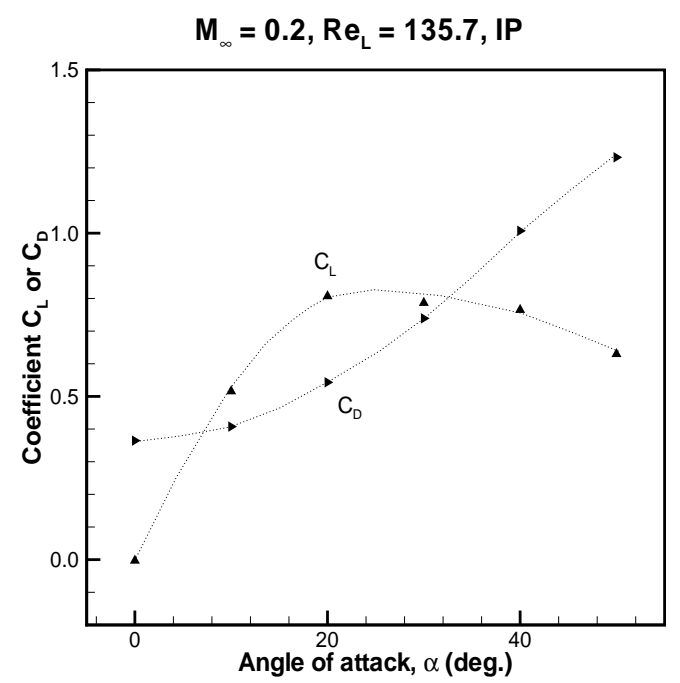

Figure 15. Drag coefficient and lift coefficient predicted by the hybrid approach when the Reynolds number is 135.7.

We also calculate the lift and drag coefficients of the 5\% flat plate under the free molecular condition using equations (9) and (10) (Gombosi, 1994), where $\alpha$ is the angle of attack and $s=\sqrt{V_{\infty}^{2} /(2 R T)}$. Figure 18 shows that the lift slope is as high as 16.5 and the drag coefficient is larger than 7.9. The ratio of lift to drag, however, is less than 1.

$$
\begin{gathered}
C_{L}=\cos \alpha\left[\frac{\operatorname{erf}(s \sin \alpha)}{s^{2}}+\sqrt{\pi} \frac{\sin \alpha}{s}\right] \\
-0.05 \sin \alpha\left[\frac{\operatorname{erf}(s \cos \alpha)}{s^{2}}+\sqrt{\pi} \frac{\cos \alpha}{s}\right] \\
C_{D}=\frac{2}{\sqrt{\pi}}\left[\frac{e^{-s^{2} \sin ^{2} \alpha}}{s}+\sqrt{\pi} \sin \alpha\left(1+\frac{1}{2 s^{2}}\right) \operatorname{erf}(s \sin \alpha)+\pi \frac{\sin ^{2} \alpha}{2 s}\right] \\
+0.05 \frac{2}{\sqrt{\pi}}\left[\frac{e^{-s^{2} \cos ^{2} \alpha}}{s}+\sqrt{\pi} \cos \alpha\left(1+\frac{1}{2 s^{2}}\right) \operatorname{erf}(s \cos \alpha)+\pi \frac{\cos ^{2} \alpha}{2 s}\right]
\end{gathered}
$$




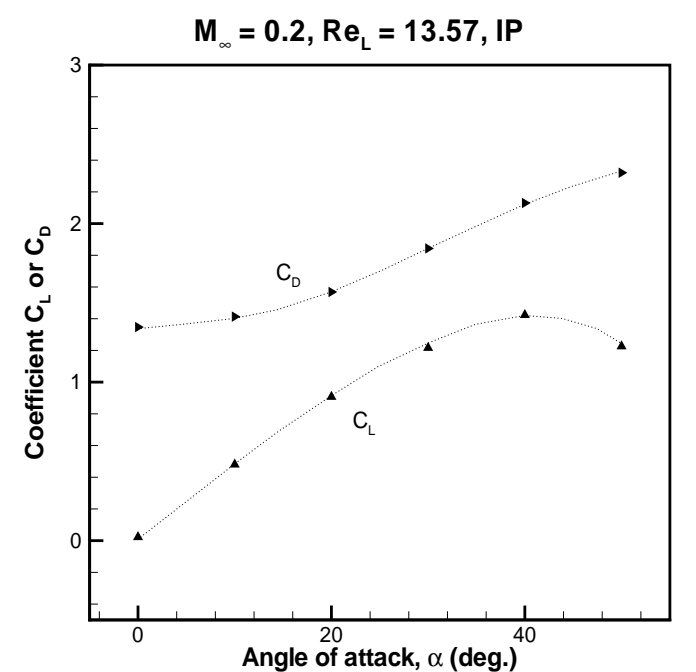

Figure 16. Drag and lift coefficients when $R e=13.57$.

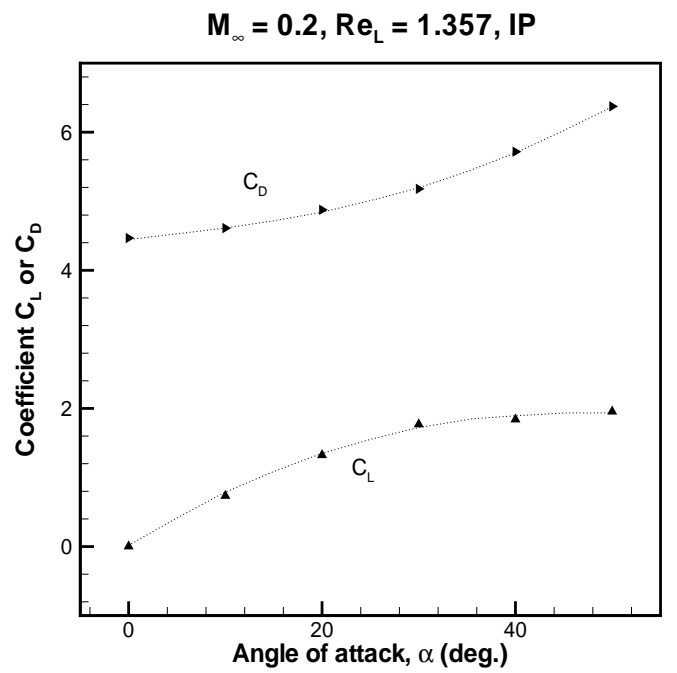

Figure 17. Drag and lift coefficients when $\mathrm{Re}=1.357$.

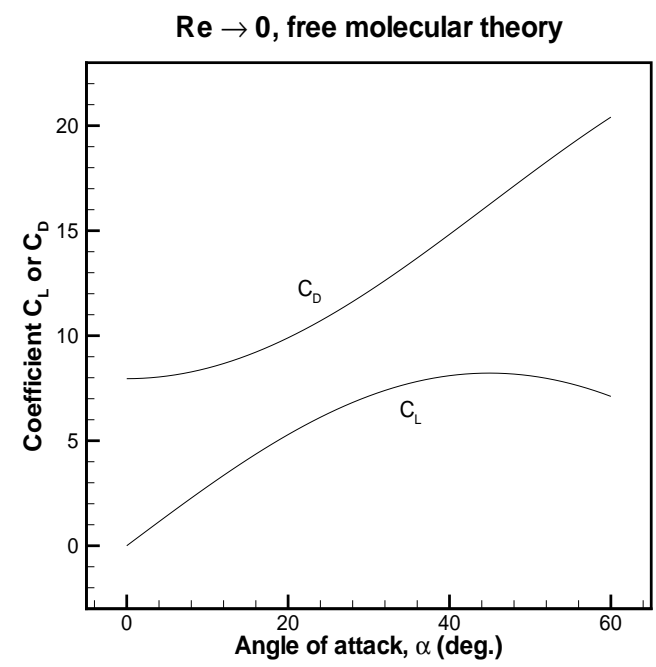

Figure 18. Drag and lift coefficients predicted by the free molecular theory.
The Reynolds number effects on the lift slope and the minimum drag coefficient of the $5 \%$ flat plate airfoil are illustrated in Figure 19. The results show that there is a minimum lift slope at a Reynolds number near 10 for the aerodynamic characteristics of the airfoil, while the drag coefficient keeps increasing when the Reynolds number decreases.

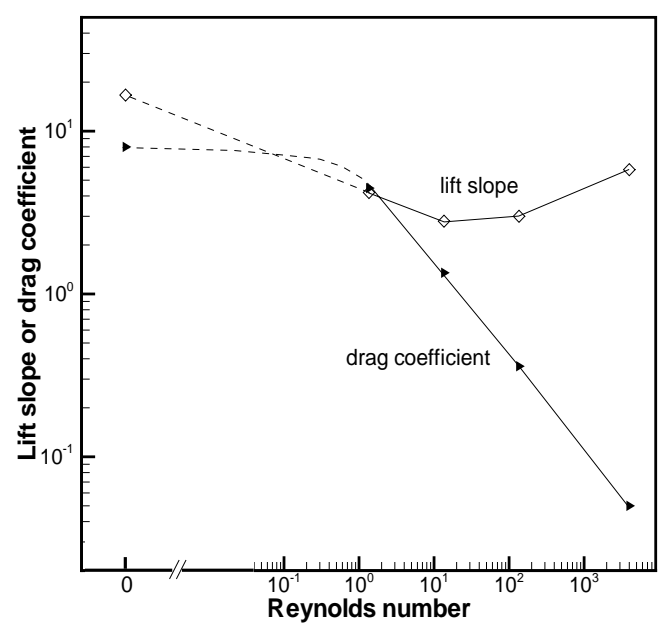

Figure 19. Reynolds number effects on the lift slope and drag coefficient of the $5 \%$ flat plate airfoil.

\section{CONCLUSIONS}

The flow over a 5\% flat plate and the aerodynamic characteristics of the flat plate airfoil at low Reynolds numbers below 1,000 were investigated using a hybrid continuumparticle approach.

Studies showed that the low Reynolds number flows are very different from high Reynolds number flows. First, the viscous force dominates low Reynolds number flows. The leading and trailing edges affect a significant fraction of the plate length. The boundary layer equations are no doubt inappropriate. The flow around the plate is severely slowed and the skin friction is very large when the Reynolds number is small. Second, low Reynolds number flows are compressible. The pressure coefficient increases when the Reynolds number decreases. The density variation can be as high as 15 percent of the density of the free stream having a Mach number of 0.2 when the Reynolds number is about 1. Hence, the incompressible assumption is not valid for low Reynolds number flows. Third, rarefied effects become important for low Reynolds number flows. Rarefied phenomena start to appear near the leading and trailing edges and expand to domains around the airfoils when the Reynolds number decreases. Therefore, continuum equations cannot describe the whole flow any more.

The aerodynamic characteristics of the $5 \%$ flat plate are poor at low Reynolds numbers. In general, the ratio of lift to drag drops below 1 when the Reynolds number is less than 50 . 
The drag coefficient monotonically increases when the Reynolds number decreases. There is a minimum lift slope of about 2.8 for the $5 \%$ flat plate airfoil at a Reynolds number near 10. However, flexible and flapping wings may increase the lift and thrust for micro air vehicles and tiny flyers in nature.

\section{ACKNOWLEDGMENTS}

The authors greatly appreciate the support from the Air Force Office of Scientific Research through MURI grant F49620-98-1-0433.

\section{References:}

Bird, G.A., 1994, "Molecular Gas Dynamics and the Direct Simulation of Gas Flows," Oxford Science Publications, New York

Fan, J., and Shen, C., 2001, "Statistical Simulation of Low-Speed Rarefied Gas Flows," Journal of Computational Physics, vol. 167, pp. 393-412

Garcia, A.L., Bell, J.B., Crutchfield, W.Y. and Alder, B.J., 1999, "Adaptive Mesh and Algorithm Refinement Using Direct Simulation Monte Carlo," Journal of Computational Physics, vol. 154, pp. 134-155

Garcia, A.L. and Alder, B.J., 1998, "Generation of the ChapmanEnskog Distribution," Journal of Computational Physics, vol. 140 , pp. 66-70

Gombosi, T.I., 1994, “Gaskinetics Theory,” Cambridge University Press, Cambridge
Hirsh, C., 1991, "Numerical Computation of Internal and External Flows, II," John Wiley \& Sons, West Sussex, England

Jones, R.T., 1990, "Wing Theory," Princeton University Press, New Jersey

MacCormack, R.W. and Candler, G.V., 1989, "The Solution of NavierStokes Equations Using Gauss-Seidel Line Relaxation," Computer \& Fluids, vol. 17, pp. 135-150

McMichael, J.M. and Francis, M.S., 1997, "Micro Air Vehicles Toward a New Dimension in Flight," DARPA, http://www.darpa.mil/tto/MAV/mav_auvsi.html

Mueller, T.J. (ed.), 2001, "Fixed and Flapping Wing Aerodynamics for Micro Air Vehicle Applications," AIAA, Inc., Reston

Schaaf, S.A. and Sherman, F.S., 1954, "Skin Friction in Slip Flow," Journal of the Aeronautical Sciences, vol. 21 (2), pp. 85-90

Schaaf, S.A. and Chambre, P.L., 1958, "Flow of Rarefied Gases," High Speed Aerodynamics and Jet Propulsion, vol. 3(H), Princeton University Press, pp. 678-739

Sun, Q., 2003, "Information Preservation Methods for Modeling Micro-Scale Gas Flows," $\mathrm{PhD}$ dissertation, University of Michigan, Ann Arbor

Sun, Q. and Boyd, I.D., 2002, "A Direct Simulation Method for Subsonic, Microscale Gas Flows," Journal of Computational Physics, vol. 179, pp. 400-425

Sun, Q., Boyd, I.D. and Candler, G.V., 2003, "A Hybrid Continuum/Particle Approach for Micro-Scale Gas Flows,"," in Rarefied Gas Dynamics, edited by A. Ketsdever and E. Muntz, (AIP Conference Proceeding vol. 663), pp. 752-759

Sunada, S. Sakaguchi, A. and Jawachi, K., 1997, "Airfoil Section Characteristics at a Low Reynolds Number," Journal of Fluids Engineering, vol. 119, pp. 129-135 